\title{
Adenosine-induced non-sustained polymorphic ventricular tachycardia
}

\author{
Romer, M ; Candinas, R
}

\begin{abstract}
Adenosine has become widely used because of its diagnostic and therapeutic value in the emergency management of arrhythimias. it produces transient heart block by slowing conduction through tile AV node and thus terminates supraventricular tachycardias that involve the atrioventricular node. Bradyarrhythmias of short duration are common side effects of the use of this drug. Premature atrial and ventricular beats have also been reported. The very short half-life and lack of serious adverse effects generally lead to the consideration that adenosine is a safe drug. We describe a 56-year-old woman with a supra ventricular tachycardia. To terminate this rhythm disorder intravenous adenosine was given. Interruption of tile supra ventricular tachycardia was followed by non-sustained polymorphic ventricular tachycardia
\end{abstract}

DOI: https://doi.org/10.1093/oxfordjournals.eurheartj.a060488

Posted at the Zurich Open Repository and Archive, University of Zurich

ZORA URL: https://doi.org/10.5167/uzh-154084

Journal Article

Published Version

Originally published at:

Romer, M; Candinas, R (1994). Adenosine-induced non-sustained polymorphic ventricular tachycardia. European Heart Journal, 15(2):281-282.

DOI: https://doi.org/10.1093/oxfordjournals.eurheartj.a060488 


\title{
Adenosine-induced non-sustained polymorphic ventricular tachycardia
}

\author{
M. ROMER AND R. CANDINAS \\ Division of Cardiology, Department of Internal Medicine. University Hospital, CH-8091 Zürich, Switzerland
}

KEY WORDS: Adenosine, proarrhythmia, polymorphic, non-sustained ventricular tachycardia.

Adenosine has become widely used because of its diagnostic and therapeutic value in the emergency management of arrhythmias. It produces transient heart block by slowing conduction through the $A V$ node and thus terminates supraventricular tachycardias that involve the atrioventricular node. Bradyarrhythmias of short duration are common side effects of the use of this drug. Premature atrial and ventricular beats have also been reported. The very short half-life and lack of serious adverse effects generally lead to the consideration that adenosine is a safe drug. We describe a 56-year-old woman with a supraventricular tachycardia. To terminate this rhythm disorder intravenous adenosine was given. Interruption of the supraventricular tachycardia was followed by non-sustained polymorphic ventricular tachycardia.

\section{Introduction}

Adenosine is a rapidly metabolized endogenous purine nucleoside that has recently become very useful for the treatment and diagnosis of cardiac arrhythmias. It is effective in terminating most supraventricular tachycardias in which the AV node forms part of the reentrant circuit. Additionally, it may unmask an underlying atrial arrhythmia by slowing AV node conduction. Due to the short half-life of adenosine its clinical side effects last less than $1 \mathrm{~min}$ and serious haemodynamic adverse effects are absent. Therefore, adenosine has become the preferred drug, compared to verapamil or beta-blockers, for terminating supraventricular tachycardias $^{[1]}$. Previously published review articles ${ }^{[2,3]}$ mentioned that short runs of non-sustained ventricular tachycardias seldom occur after administration of adenosine. To our knowledge, only one study originally reported this rare arrhythmic phenomenon but did not give a detailed description ${ }^{[4]}$. In the present case we describe the occurrence of a polymorphic non-sustained ventricular tachycardia after adenosine was given to terminate an episode of supraventricular tachycardia.

\section{Case report}

A 56-year-old woman was admitted because of a recurrence of a paroxysmal supraventricular tachycardia. The ECG recording on several occasions between tachycardia episodes had always showed sinus rhythm with normal PR and QTc intervals. Drug therapy comprised verapamil $40 \mathrm{mg}$ once daily and phenobarbital $20 \mathrm{mg}$. On admission, the patient complained of

Submitted for publication on 23 June 1993.

Correspondence. Markus Romer, MD, Division of Cardiology, Department of Internal Medicine, Unıversity Hospital, Rāmistrasse 100, CH-8091 Zünch, Switzerland. chest tightness and palpitations. Blood pressure was $110 / 80 \mathrm{mmHg}$, heart rate 180 beats $\min ^{-1}$, and physical examination showed no evidence of heart failure. The ECG indicated the presence of AV nodal reentrant tachycardia, and since the vagal manoeuvres failed to terminate the tachycardia, adenosine was given as a $6 \mathrm{mg}$ i.v. bolus. Immediately after successful termination of the supraventricular tachycardia, a polymorphic non-sustained ventricular tachycardia of 10 complexes ( 180 beats . $\min ^{-1}$ ) occurred (Fig. 1). Simultaneously the patient complained of transient lightheadedness and chest discomfort. Blood pressure was not monitored. After resolution of the tachycardia the repeated 12-lead ECG and a two-dimensional echoDoppler study showed no abnormality. Routine laboratory tests were within normal limits. Neither a bicycle exercise test nor an ambulatory electrocardiogram demonstrated any ventricular arrhythmias. The patient refused to be rechallenged with another bolus of adenosine while in stable sinus rhythm.

\section{Discussion}

Sinus bradycardia and bradyarrhythmias (transient sinus arrest, complete heart block or asystole) may follow adenosine administration. Premature isolated atrial and ventricular complexes are often observed after adenosine-induced termination of tachycardias ${ }^{\text {II }}$. The rare occurrence of short runs of ventricular tachycardias has been quoted ${ }^{[2,3]}$, but no serious consequences of this proarrhythmic effect have been reported ${ }^{[4]}$. Despite extensive research on the cardiac and cardiovascular effects of adenosine $\mathrm{e}^{[3]}$, the pathophysiological mechanism of the premature ventricular contractions that develop after adenosine-induced termination of a supraventricular tachycardia remains unknown. A direct myocardial proarrhythmic effect seems unlikely because, in contrast to what is observed 


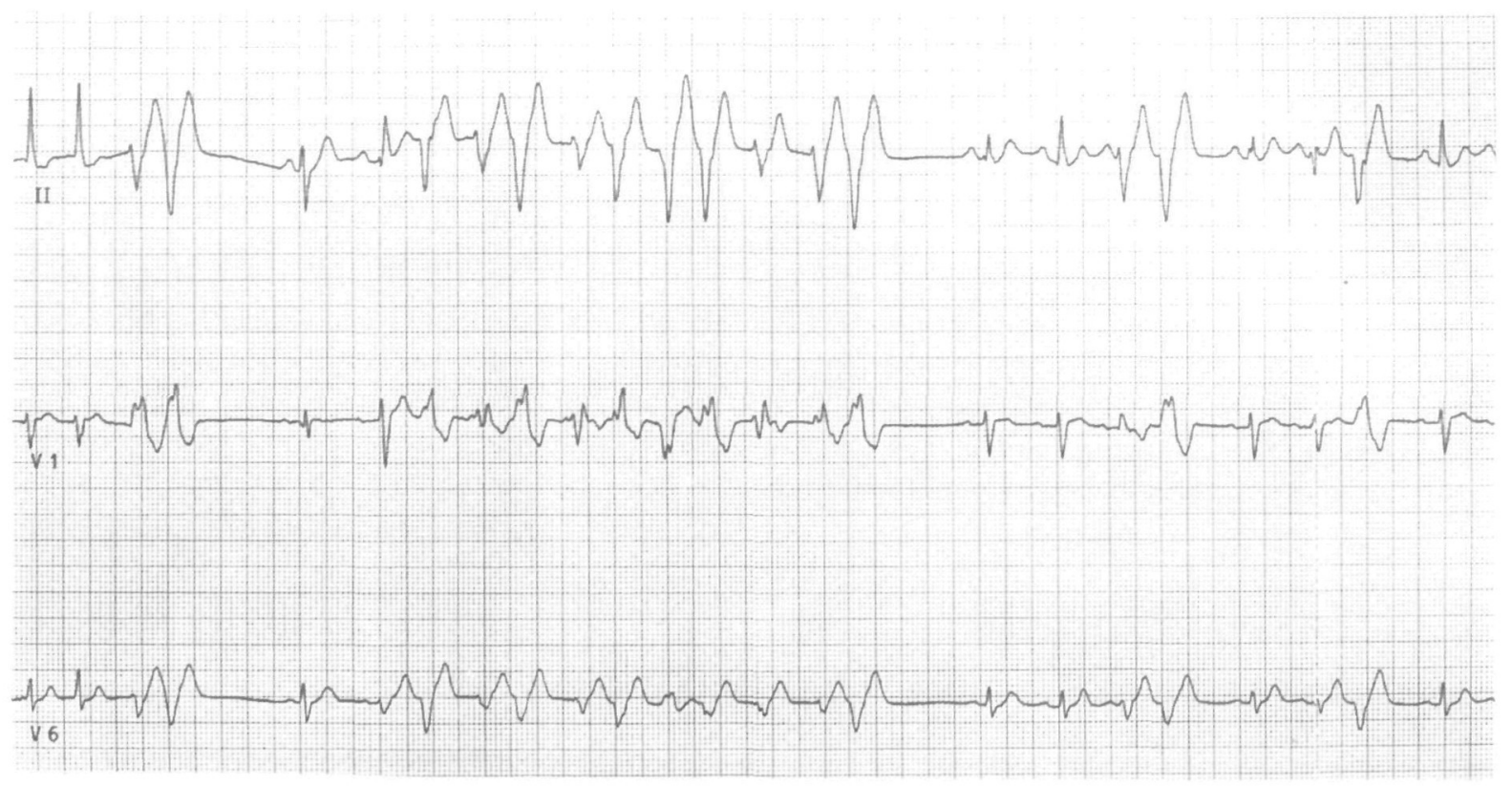

Figure 1 Polymorphic non-sustained ventricular tachycardia following the termination of the supraventricular tachycardia after adenosine was given. Paper speed is $25 \mathrm{~mm} . \mathrm{s}^{-1}$.

in supraventricular tissue, no direct effect of adenosine (e.g. receptor-mediated activation of potassium channels) has been observed in ventricular myocytes ${ }^{[3]}$. However, adenosine activates autonomic reflex mechanism (by chemoreceptor stimulation) and increases sympathetic ton $\mathrm{e}^{[5]}$. Additional factors, such as the unpleasant side effects of adenosine and the preceding supraventricular tachycardia may contribute to raising catecholamines levels and facilitating runs of ventricular extrasystole.

\section{Conclusion}

It is known that runs of ventricular premature beats can initiate more severe and even fatal ventricular tachyarrhythmias, especially in patients with organic heart disease. Whatever may be the underlying mechanisms of adenosine-induced ventricular extrasystole, our case illustrates a serious proarrhythmic effect of adenosine. It has to be emphasized that potentially lethal tachyarrhythmias may follow intravenous adenosine. Thus, instruments for resuscitation, including a DC-cardioverter, are mandatory when this drug is administered.

\section{References}

[1] Camm AJ, Garratt CJ. Review article: Adenosine and supraventricular tachycardia. N Engl J Med 1991; 325: 1621-9.

[2] Rankin AC, Brooks R, Ruskın JN, McGovern BA. Adenosine and the treatment of supraventricular tachycardia. Am J Med 1992; 92: 655-64.

[3] Lerman BB, Belardinelli L. Cardiac electrophysılogy of adenosine. Circulation 1991; 83: 1499-509.

[4] Rankin AC, Oldroyd KG, Chong E, Rae AP, Cobbe SM. Value and limitations of adenosine in the diagnosis and treatment of narrow and broad complex tachycardias. Br Heart $\mathrm{J}$ 1989; 62: 195-203.

[5] Biaggioni I, Olafsson B, Robertson RM, Hollister AS, Robertson D. Cardiovascular and respiratory effects of adenosine in conscious man. Circ Res 1987; 61: 779-86. 